\title{
La doble redacción en el Ars grammatica de Julián de Toledo*
}

\author{
José Carracedo-Fraga \\ Universidad de Santiago de Compostela \\ jose.carracedo@usc.es \\ ORCID iD: https://orcid.org/0000-0002-6645-7156
}

The Double Version in Julian of Toledo's Ars Grammatica

El Ars grammatica compuesta por Julián de Toledo probablemente entre los años 680-690 fue muy pronto objeto de varias modificaciones intencionadas en las dos ramas de transmisión textual de la obra. Las variaciones afectaron a la explicación de algunos contenidos, a la selección de ejemplos ilustrativos y a la utilización de citas de auctores. Los responsables de esas intervenciones trabajaban en alguna escuela visigótica muy próxima a la del propio Julián, ya que utilizaron los mismos materiales y siguieron el mismo estilo de redacción que el maestro toledano. Por lo tanto, las dos versiones derivan de forma más o menos directa de las enseñanzas de Julián.

Palabras clave: gramática; Julián de Toledo; doble redacción.
The Ars grammatica composed by Julian of Toledo probably between the years 680-690 was very soon the object of several deliberate modifications in the two branches of the textual transmission of the work. Variations affected the explanation of some contents, the selection of illustrative examples, and the use of quotes from auctores. The authors of these interventions worked in a Visigothic school very close to Julian's own, since they used the same materials and followed the same writing style as the teacher from Toledo. Therefore the two versions derive more or less directly from Julian's teachings.

Key words: grammar; Julian of Toledo; double version.

Cómo citar este artículo / Citation: Carracedo-Fraga, José (2021): «La doble redacción en el Ars grammatica de Julián de Toledo», Emerita 89 (1), pp. 127-148.

* Este trabajo fue elaborado en el marco del proyecto de investigación «Textos técnicos latinos: medicina y gramática entre la Antigüedad tardía y la alta Edad Media», financiado por el Ministerio de Ciencia, Innovación y Universidades (PGC2018-093580-B-I00). Agradezco las atinadas y útiles observaciones de los evaluadores del trabajo. 


\section{Introducción}

Cualquier texto transmitido de generación en generación por vía oral o por vía escrita puede ser sometido a diverso tipo de intervenciones o manipulaciones. Cuando además los textos ofrecen saberes útiles, es decir, contenidos técnicos y prácticos, esos textos son por antonomasia de carácter abierto. Y si a todo lo anterior añadimos que los textos son gramaticales y, por lo tanto, destinados a su uso en la escuela, su carácter abierto queda todavía mucho más acentuado. Un maestro de gramática de la Antigüedad o de la Edad Media pone por escrito en un momento dado un estado de enseñanza concreto, pero ese programa didáctico particular es necesariamente el resultado de adaptar a su momento toda una tradición anterior y a su vez ese resultado puede ser susceptible de intervenciones por parte de lectores o de otros maestros posteriores para ser acomodado a un nuevo momento y a unas nuevas circunstancias. Con toda razón definía Donato la ciencia gramatical antigua como un munus collaticium ${ }^{1}$, esto es, como el fruto de la aportación sucesiva y acumulativa de saberes e interpretaciones de maestros que van adaptando sus enseñanzas a sus propios discípulos.

A pesar de la dificultad que siempre supone definir con claridad momentos y protagonistas en ese proceso sucesivo de intervenciones en los textos abiertos, en este trabajo yo pretendo hacer visibles algunos aspectos de cómo afectó ese proceso de reelaboración al tratado gramatical debido a Julián de Toledo incluso ya en los primeros estadios de su existencia. Para ello es necesario comenzar recordando algunos datos que permitan situar el origen del Ars grammatica que nos ocupa y dibujar a grandes rasgos sus caminos de difusión.

\section{Un producto de la escuela visigótica}

Desde que esa Ars grammatica fue recuperada para el mundo moderno gracias a la edición del cardenal toledano Francisco de Lorenzana en el año 1797 dentro de su ambicioso proyecto de edición de la obra de todos los Padres de

\footnotetext{
1 Don., Epist. 7.
} 
Toledo 2 , el manual de gramática quedó asociado a Julián de Toledo (ca. 644690), porque a este autor va atribuido el tratado en el manuscrito vaticano conocido y utilizado por el editor, el códice Pal. Lat. 1746. Desde entonces ha habido teorías a favor o en contra de esa autoría, pero casi siempre ha habido más unanimidad sobre el carácter visigótico de la obra ${ }^{3}$. Repaso brevemente los principales argumentos que sirven para considerar que el tratado gramatical es el resultado de las enseñanzas en un centro escolar de la Hispania visigótica:

$1^{\circ}$ ) Aparecen mencionados expresamente en ejemplos gramaticales los reyes visigodos Ervigio (24, 379 y 40, 153) y Égica (171, 22 Munzi) ${ }^{4}, y$ también varios otros nombres de personajes clara o probablemente visigóticos: Afrila (122, 206 y 130, 77), Donumdei (40, 151), Gisclamundo (23, $346)$, Isidoro $(18,221 ; 23,356 ; 34,17)$, Onemundo $(23,355)$, Romarico (23, 341), Trasemiro (172, 1 Munzi), Trasemundo (171, 23 Munzi), Vincila (172, 13 Munzi y 179, 9 Munzi).

$2^{\circ}$ ) Son utilizados nombres de ciudades hispanas para ejemplificar usos gramaticales de lugares: Mérida $(88,225)$, Cartagena $(88,226)$, Zaragoza $(88,239)$, Barcelona $(88,242)$ y, sobre todo, Toledo en tres ocasiones $(88$, $228 ; 88,238 ; 88,241)$.

$3^{\circ}$ ) Hay otras alusiones a elementos característicos visigóticos, como la significativa referencia expresa al nombre del llamado Liber Commicus Toletanus $(178,33)^{5}$.

${ }^{2}$ Lorenzana 1797. Una copia manuscrita del códice vaticano realizada por encargo del cardenal Lorenzana se conserva con la signatura 119 en la colección Borbón-Lorenzana de la Biblioteca de Castilla-La Mancha en Toledo.

${ }^{3}$ Sobre ello se discutía ya a principios del siglo XX en trabajos como los de Funaioli 1911 y Beeson 1924. Revisiones más recientes pueden verse en Carracedo-Fraga 2005b y 2015, pp. 9-33.

${ }^{4}$ Para todos los textos gramaticales que cito en este trabajo utilizo el sistema de mencionar página y (separadas por coma) líneas de la edición utilizada. En el caso del Ars grammatica de Julián remito a la edición de Maestre Yenes 1973 y para la parte relativa a la sección De partibus orationis II a la edición de Munzi 1980-1981. Argumentos a favor de que esta última sección forma parte del conjunto gramatical están analizados en el trabajo de Munzi y también más recientemente en Carracedo-Fraga 2018.

${ }_{5}^{5}$ Sobre el Liber Commicus véase el trabajo de Pérez de Urbel y González y RuizZorrilla 1950. 
$4^{\circ}$ ) La versión del texto de las Artes del gramático romano Elio Donato (fl. ca. 350) utilizada corresponde a la conservada por la considerada familia visigótica de transmisión de la obra del gramático romano ${ }^{6}$.

$5^{\circ}$ ) Varias de las citas bíblicas reproducidas presentan un texto coincidente con versiones de las primitivas traducciones al latín de la Biblia conocidas como la Vetus Latina que son características de la Hispania visigoda, especialmente en lo relativo al libro de los Salmos ${ }^{7}$.

$\left.6^{\circ}\right)$ Entre los numerosos auctores citados como modelo de lengua se concede un gran protagonismo a poetas cristianos leídos y apreciados en la Hispania visigótica (Ausonio, Coripo, Draconcio, Prudencio, Sedulio, Venancio Fortunato) y de forma especial a poetas visigóticos, como Isidoro de Sevilla, Eugenio de Toledo o el anónimo autor del singular Epitaphium Antoninae. También ocupan un lugar destacado varios textos propios de la liturgia visigótica, principalmente himnos ${ }^{8}$.

$7^{\circ}$ ) La propia tradición textual del Ars grammatica remonta seguramente a un antecedente común copiado en letra visigótica. Así lo dan a entender errores de lectura atribuibles a ese arquetipo y explicables a partir de una mala interpretación de abreviaturas o grafías propias del sistema de escritura visigótica. También apoya esa teoría la pervivencia en la tradición textual de abreviaturas peculiares de esa escritura peninsular, a pesar de que todos los códices que hoy conservamos y conocemos fueron copiados fuera de la Península y en otros tipos de escritura. Añádase que el Ars que nos ocupa circuló formando parte de compilaciones gramaticales probablemente debidas a la escuela visigótica, como las transmitidas por los códices Bern 207 y Paris lat. $7530^{9}$.

Las referencias a personajes visigóticos de la corte ${ }^{10}$, la particular querencia por la ciudad de Toledo y la especial relación con la escuela poética toledana son indicios que sirven para situar el origen del tratado gramatical en la

${ }^{6}$ Ha podido definir claramente esa familia Holtz 1981, pp. 453-474.

7 Véase Carracedo-Fraga 2014b.

${ }^{8}$ Están allí citados una o varias veces versos de los himnos de la liturgia hispana recogidos bajo los números 104, 133, 137, 187, 189, 204 y 209 en la colección editada por Castro Sánchez 2010. Sobre poetas leídos en la escuela visigótica escribe Carracedo-Fraga (2005a), y sobre la asociación de poesía y gramática visigóticas y su influencia en la escuela carolingia trata Alberto (2016 y 2017).

${ }^{9}$ Véase Holtz 1981, pp. 361-364 y 454-455.

${ }^{10}$ El reinado de Ervigio se desarrolla en los años 680-687 y el de Égica en los años 687-702. Por otra parte, personajes con algunos de los nombres citados en el Ars gramma- 
capital del reino visigodo en el último cuarto del siglo VII. En ese momento la gran figura literaria y teológica es Julián, obispo de la sede toledana entre los años 680 y 690, el cual en ningún momento dejó de ejercer su labor de magisterio. A Julián aparece atribuido el manual de gramática en uno de los manuscritos principales que transmiten la obra, el ya mencionado códice $P a l$. Lat. 1746, copiado a finales del siglo VIII y principios del siglo IX en la abadía de San Nazario de Lorsch. También quedan noticias de atribución a Julián en catálogos de códices de las bibliotecas de las abadías de Saint-Riquier y de Fulda ${ }^{11}$. Por otra parte, hay muy notables paralelismos, sobre todo, de contenido, pero también de lengua, estilo y uso de fuentes entre el Ars grammatica y otras obras de Julián que arrojan algo de luz sobre la cuestión de la autoría.

\section{La tradición textual de la obra}

Por lo que hoy conocemos, estos son los manuscritos medievales que transmiten directamente el tratado gramatical completo o algunas de sus partes ${ }^{12}$ : $B=$ Berna, Burgerbibliothek, 123, s. IX ${ }^{\text {med }}$, Fleury, ff. $117 \mathrm{r}-128 \mathrm{v}$, transmite solo el inicio del tratado, ya que acaba trunco en el capítulo De uerbo (59, 203) por pérdida de folios finales.

$E=$ Erfurt, Universitätsbibliothek, $C A 2^{\circ} 10$, s. IX ${ }^{\text {in }}$, región de Austrasia, ff. $1 \mathrm{r}-44 \mathrm{r}, 60 \mathrm{v}-69 \mathrm{v}$ y $121 \mathrm{r}-122 \mathrm{r}$, transmite toda la obra salvo la Conlatio de generibus metrorum y la sección De partibus orationis II.

$F=$ Berna, Burgerbibliothek, 207, s. VIII ${ }^{\text {ex }}$, Fleury, ff. $18 \mathrm{v}-77 \mathrm{v}$ y $81 \mathrm{v}-$ 101r, es el único códice que transmite el tratado completo.

tica aparecen firmando en las actas de los concilios XIII, XIV y XV de Toledo, celebrados respectivamente en los años 683, 684 y 688.

${ }^{11}$ El catálogo de Saint-Riquier del año 831 puede leerse en Becker 1885, p. 25. El catálogo de Fulda es del siglo XVI y está transcrito en Christ 1933, pp. 151-152 y 235-236.

12 Para una presentación más completa de los códices véase Maestre Yenes 1973, pp. LXI-LXXV, y Carracedo-Fraga 2015, pp. 81-102. También transmitía el Ars grammatica en una versión muy próxima a la del ejemplar de Erfurt la primera sección del códice de la Bibliothèque Municipale de Chartres 92 (47), s. IX, destruido durante la Segunda Guerra Mundial. El códice aparece descrito en el t. 11 del Catalogue général des manuscrits des bibliothèques publiques de France, pp. 48-49, y fue consultado en su momento por Beeson 1924, p. 60. También está copiado un muy pequeño fragmento sobre el capítulo de littera en el códice de Múnich CLM 807, ff. 71r-v, s. XV. 
$G=$ Gotha, Forschungsbibliothek, Memb. II 193, s. VIII , ¿Fulda?, 2 ff., es un bifolio utilizado como guarda de otro códice, que conserva la parte final del capítulo De tropis (desde 211,240) y el inicio de la Conlatio de generibus metrorum (hasta 222, 10).

$L=$ Ciudad del Vaticano, Biblioteca Apostolica Vaticana, Pal. Lat. 1746, s. VIII ${ }^{\text {ex }}$ (ff. 1r-10v y 72r-126r, para esta sección utilizo la sigla $L_{1}$ ) y s. IX ${ }^{\text {in }}$ (ff. 11r-71r y 126v-184v, para esta sección utilizo la sigla $L_{2}$ ), Lorsch, ff. 72r$98 \mathrm{v}$ y $126 \mathrm{v}-152 \mathrm{r}$, es otro de los códices que transmite casi todo el conjunto, ya que solamente falta el apartado De partibus orationis $I I^{13}$.

$N=$ Nápoles, Biblioteca Nazionale, IV.A.34, s. IX ${ }^{\text {in }}$ Luxeuil, ff. 266v-271r y $272 \mathrm{v}-273 \mathrm{r}$, contiene la sección De uitiis et uirtutibus orationis, trunca en su parte final (termina en 205, 67).

$P=$ París, Bibliothèque Nationale de France, lat. 18520, s. IX ${ }^{2 / 4}$, región de París, ff. $127 \mathrm{v}-135 \mathrm{v}$, conserva una selección de capítulos del tratado gramatical: De littera (113, 1-126, 277), De accentibus y De posituris (170, 1-178, 35), De barbarismo, De soloecismo y De ceteris uitiis (179, 1-188, 40; finaliza ex abrupto por la pérdida de algunos folios).

$R=$ Ciudad del Vaticano, Biblioteca Apostolica Vaticana, Reg. Lat. 1586, s. IX ${ }^{3 / 4}$, Reims, ff. 73r-77r, dentro de un conjunto de textos métricos incluye la Conlatio de generibus metrorum.

$T=$ París, Bibliothèque Nationale de France, lat. 7540, s. IX ${ }^{\text {med }}$, Lyon, ff. 49r$52 \mathrm{v}$ y $60 \mathrm{r}-60 \mathrm{v}$, ofrece un extracto del capítulo sobre el adverbio $(79,1-87,215)$. $V=$ Valenciennes, Médiathèque Simone Veil, 393 (376), s. IX ${ }^{1 / 3}$, región del Loira, ff. 152r-155r, transmite también exclusivamente la Conlatio ${ }^{14}$.

Del elenco de testimonios directos conservados podemos deducir que el Ars grammatica de Julián circuló principalmente en los siglos VIII y IX por centros de estudio carolingios de la Galia y del oeste de la Germania, algunos de ellos habitados por eruditos insulares (irlandeses o anglosajones) o de formación insular, los cuales dejaron en el proceso de transmisión numerosas señales de su propio sistema de escritura. Un papel importante lo jugaron, sin duda, el obispo de Orléans de origen visigodo Teodulfo ( $\uparrow$ 821) y su círculo intelectual.

13 Tal como se explica más arriba en la nota 2, el texto de este manuscrito fue utilizado por Lorenzana para su edición de 1797 y de la parte correspondiente al tratado gramatical de Julián hizo sacar una copia manuscrita el arzobispo toledano.

${ }^{14}$ Este testimonio ha sido recientemente puesto en escena por Alberto 2018. 
La comparación del texto transmitido por los diferentes manuscritos antes presentados permite establecer que el Ars grammatica siguió en un momento cercano a la fecha de redacción dos caminos de transmisión diferentes ${ }^{15}$. Además de las variaciones de redacción que comentaremos más adelante, numerosas variantes textuales separativas debidas a errores de copia permiten establecer dos subarquetipos a los que llamamos $\alpha$ y $\beta$. A la primera familia pertenecen los códices $F, G, L_{l}, N, P, R, T$ y $V$, y de la segunda rama de transmisión forman parte los testimonios $B, E$ y $L_{2}{ }^{16}$. Sirva como muestra probatoria la siguiente pequeña selección de variantes extraídas del inicio del Ars grammatica:

10,21 inchoauit a nomine $\alpha$ : a nomine inchoauit $\beta$

10,25 hominem $\alpha$ : hodie $\beta$

11,63 se non iunxit $\alpha$ : non seiunxit $\beta$

12,88 aut quislibet $\alpha$ : si qualislibet $\beta$

13,93 adnumerabit $\alpha$ : denumerauit (dinum- $\left.E^{a c} L\right) \beta$

14,135 qui - mihi om. $\beta$ (por salto de igual a igual)

16,170 praepositiuo $\alpha$ : positiuo $\beta$

19, 258 quia - dicta om. $\alpha$ (por salto de igual a igual)

22,337 da simile $\alpha$ : passibile $\beta$

23,356 pluribus $\alpha$ : plurimis $\beta$

Varios errores textuales compartidos por las dos familias $\alpha$ y $\beta$, que suponemos que no son responsabilidad del compilador del tratado gramatical, sino que fueron debidos a fallos en el proceso de transmisión manuscrita, inducen a reconstruir un arquetipo $\mathrm{X}$ del que derivan esos errores comunes. Estos son algunos de esos errores de copia debidos a omisiones por haplografía o por salto de igual a igual, a confusiones de letras, palabras o abreviaturas, o a transposiciones:

${ }^{15}$ Lo que aquí comento de forma breve sobre el proceso de transmisión de la obra como resultado de una colación personal de los testimonios puede ser comparado y completado con lo que explican al respecto Maestre Yenes 1973, pp. LXXXV-CV, y Carracedo-Fraga 2015, pp. 102-124. Evidentemente, respecto al trabajo de la primera incluyo testimonios no utilizados por ella y respecto al estudio del segundo tengo en cuenta el conjunto del tratado gramatical.

${ }^{16}$ Recuérdese que el manuscrito de Lorsch fue copiado en dos momentos distintos: las manos más antiguas $\left(L_{l}\right)$ copian toda la segunda parte del Ars desde el capítulo de littera; a las manos más recientes $\left(L_{2}\right)$ se debe la primera sección sobre las partes orationis. 
72, 551-552 Quae in 'o' desinunt et ex se accepta ' $r$ ' littera faciunt passiua, ut 'lego, legor'.

et ex se coll. Don. ,min. 592, 16-17 correxi : ex se et codd.

75, 633-634 Ex pluribus, ut 'adoleo'. <Quomodo? 'A' praepositio est>, 'do' uerbum est, 'leo' nomen est.

Quomodo - est suppleui

97, 67-69 'Aut' est coniunctio, est et aduerbium: quando pro 'non' fuerit positum ... pro non correxi : pronomen codd.

104, 28 In contrarium uertit, <ut 'felix', 'infelix', aut nihil significat>, ut 'natat', 'innatat'.

ut - significat coll. Explan. in Don. 517, 9-10 suppleui

184, 45-47 Sicut «Hauriat hunc oculis ignem crudelis ab alto Dardanus»; $<$ 'Dardanus'> enim pro 'Dardanius', proprium pro appellatiuo posuit.

Dardanus suppl. Maestre

187, 8-9 Et illud: «Tumida ex ira tum corda resident».

ira tum corda coll. Verg., Aen. VI 407 correxi: iurato corde FN, iuratio corde $P$, irato corde $E L$

198, 87-88 «Ecce Dionaei processit Caesaris astrum, astrum quo segetes gauderent frugibus»

astrum astrum ... gauderent coll. Verg., ecl. IX 47-48 correxi : antrum antrum ... gaudet et codd.

200, 139-140 «Eos reduci quam relinqui, deuehi quam deseri malui».

relinqui deuehi coll. Enn., trag. 392-393 corr. Maestre : relinquere uei $F$, relinquere uehi $L_{1} N$, relinqui reu hei $E$

214, 292 Constituta tamen inter incommutabilem supra se ueritatem et mutabilia infra se cetera.

se ueritatem coll. Aug., doctr. Chr. II 38. 57 correxi : seruitutem codd.

Teniendo en cuenta lo anterior y tomando en consideración todas las relaciones que pueden ser establecidas entre los distintos testimonios manuscritos a partir de errores textuales o incluso a partir de rasgos de elementos paratextuales conjuntivos y separativos, el proceso de transmisión directa por nosotros conocida puede ser representado esquemáticamente en el siguiente stemma ${ }^{17}$ :

${ }^{17}$ Elementos paratextuales que aportan datos de relación son, por ejemplo, los tituli de las diferentes partes o la forma de introducir el diálogo entre maestro y discípulo propio del método catequístico de preguntas y respuestas que utiliza Julián. Puesto que aquí nos interesa solamente la parte alta del estema, para evitar alargar excesivamente este trabajo, omito ahora el resto de la justificación del parentesco textual entre las diferentes copias manuscritas; pue- 


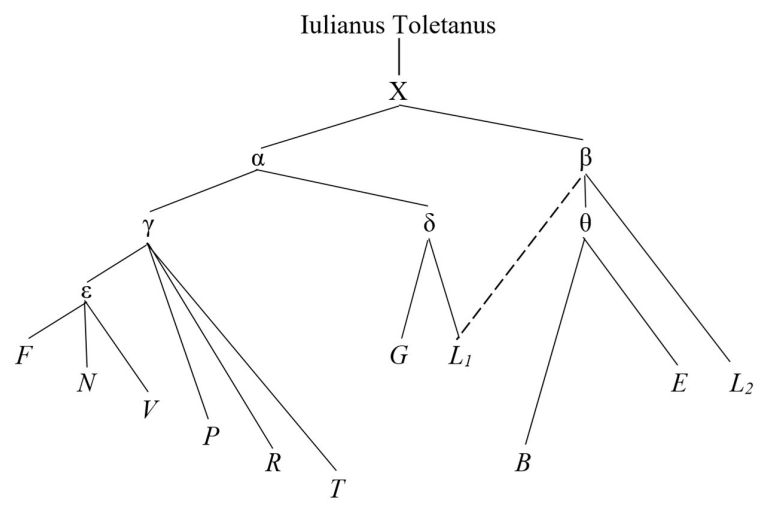

Como he comentado más arriba, la escuela carolingia jugó un importante papel en la difusión del Ars grammatica de Julián. Pero es oportuno llamar la atención sobre el hecho de que por algunos centros circularon en paralelo ejemplares de las dos ramas principales de transmisión de la obra, como lo demuestran los siguientes datos:

- A Fleury pertenecieron y casi con seguridad allí fueron copiados en momentos próximos los códices $F$ y $B$, descendientes respectivamente de los antígrafos $\alpha$ y $\beta^{18}$.

- Los copistas que trabajaron en la abadía de Lorsch en la sección más antigua del códice $L$ utilizaron un ejemplar de la familia $\alpha$ como modelo para la parte que copiaron del Ars grammatica de Julián, aunque parece que también tuvieron a su disposición y consultaron una copia perteneciente a la familia $\beta$, ya que por veces incorporaron variantes propias de esta última familia. Por su parte, los amanuenses que completaron unos años más tarde la transcripción del tratado gramatical toledano utilizaron exclusivamente como modelo un ejemplar de la familia $\beta^{19}$.

den verse algunos datos en los estudios antes citados: Maestre Yenes 1973, pp. LXXV-XCIX, Carracedo-Fraga 2015, pp. 102-112, y para el códice V Alberto 2018, pp. 180-183.

${ }_{18}$ Sirva de referencia para la relación de los dos códices con el escritorio de Fleury el trabajo de Mostert 1989, especialmente p. 58.

${ }_{19}$ Sobre la abadía de Lorsch sigue siendo un estudio de referencia Bischoff 1989; para nuestro códice vaticano véanse allí pp. 32 y 130-131. 
- En el centro de estudio de la corte de Carlomagno o en un centro muy relacionado con aquel, donde fue copiado el códice $E^{20}$ a partir de un ejemplar de la familia $\alpha$, muy poco después un lector añadió en ese mismo manuscrito en el inicio del Ars grammatica algunas correcciones textuales sirviéndose de un modelo que pertenecía a la rama $\beta$. Las intervenciones son del estilo de las siguientes:

12, 73 ut - deus sup. l. add. E2 cum $\alpha$, om. $\beta$

12, 74 ut homines sup. l. add. E E $^{2}$ cum $\alpha$, om. $\beta$

12,84 ipsud nomen sup. l. add. $E^{2}$ cum $\alpha$, om. $\beta$

12,88 aut quislibet sup. l. add. $E^{2}$ cum $\alpha$ : si qualislibet $\beta$

12, 89 reprehenditur - donatus marg. add. E2 cum $\alpha$, om. B

\section{La doble redacción del Ars grammatica}

Abundantes variaciones en el texto de la gramática toledana entre las ramas de transmisión $\alpha$ y $\beta$ difícilmente pueden ser debidas a errores en el proceso de copia y más bien tienen que ser el resultado de la intervención voluntaria de algún lector o maestro, el cual busca ofrecer en muchos pasajes una redacción alternativa diferente. Las intervenciones en el texto son de muy diverso tipo y van desde el cambio en alguna palabra hasta una amplia y reelaborada propuesta distinta, como vamos a intentar poner de manifiesto en las líneas que siguen.

Uno de los aspectos cultivados con especial atención desde la Antigüedad en la escuela del gramático fue la adquisición de vocabulario mediante ejercicios basados en la sinonimia y en las diferencias. El interés por ese tipo de ejercicios estuvo particularmente activo en las escuelas visigóticas en su objetivo de fomentar el aprendizaje de léxico amplio, variado y preciso de una lengua culta cada vez más diferenciada de la lengua hablada ${ }^{21}$. Así pues, no es nada extraño que, cuando un maestro quiere ofrecer variaciones, uno de

${ }^{20}$ Véase Bischoff 1998, p. 249.

${ }^{21}$ En el manual de gramática de Julián quedan abundantes elementos de ese interés por las differentiae y los synonyma, dos categorías gramaticales que precisamente sirvieron de base a Isidoro de Sevilla para la creación de dos de sus obras más exitosas. Sobre la presencia de esos temas en la escuela del gramático véase el resumen de Zetzel 2018, pp. 104-106; sobre el caso particular de Isidoro tratan Carracedo-Fraga (2020) y Spevak (2020, pp. LXVII-LXXII). 
los recursos que utilice sea el juego de la sinonimia en palabras y expresiones en cualquier parte del texto, pero sobre todo en fórmulas de introducción o presentación de explicaciones ${ }^{22}$ :

12,76 uocatur $\alpha$ : dicitur $\beta$

22,332 commanet $\alpha$ : habitat $\beta$

23, 344 quid est $\alpha$ : quid intellegitur $\beta$

27, 440 sicut Euangelista dicit $\alpha$ : unde et Euangelista dicit $\beta$

37,80 quomodo et pro femina $\alpha$ : sicut et pro muliere $\beta$

39,135 da eius exemplum $\alpha$ : ut puta $\beta$

49,334 quare dicuntur subiunctiua $\alpha$ : quomodo dicuntur subiunctiua $\beta$

57,169 quomodo ponuntur $\alpha:$ qualem ordinem obtinent $\beta$

63,318 unde hoc scis $\alpha$ : quomodo $\beta$

76,644 quod ad agendum pertinet $\alpha:$ quod ad futurum sit $\beta$

El Ars de Julián pertenece al grupo de los considerados manuales de gramática avanzados, es decir, aquellos tratados que buscan ofrecer un tratamiento elevado y pormenorizado de toda la teoría gramatical tradicional, principalmente mediante el comentario exegético detallado de los manuales de referencia de Donato. Sin embargo, el autor toledano, en su afán por ofrecer un manual completo, introduce también en su obra paradigmas o catálogos de flexión y análisis morfológicos más propios de tratados elementales o introductorios conocidos como Declinationes nominum o similares ${ }^{23}$. A ese afán de repaso de conocimientos de morfología responde, por ejemplo, la utilización de formas verbales variadas, jugando con la correspondiente flexión:

10,31 loquebamur $\alpha$ : locuti fueramus $\beta$

13,105 dictum est $\alpha$ : dixit (dicit $\left.L_{2}\right) \beta$

27,447 fecisti $\alpha$ : facis $\beta$

27, 439 uidebimur $\alpha$ : uidemur $\beta$

27, 457 terminatur $\alpha$ : terminari (terminare $B$ ) debetur $\beta$

34,16 ac si dicam $\alpha$ : sicut si dicas mihi $\beta$

22 Téngase en cuenta que, salvo indicación en contrario, voy a citar solamente un muy pequeño grupo de ejemplos ilustrativos de los muy numerosos existentes y por norma general ejemplos sacados del inicio del Ars grammatica.

${ }^{23}$ Sigo terminología y clasificación utilizadas por Irvine 1994, pp. 56-57, y Law 1997, especialmente pp. 54-68. 
También contribuye a ampliar el caudal de vocabulario y a la vez de contenidos la presentación de ejemplos gramaticales alternativos o en listas más completas, en algunos casos poniendo a contribución lo nuevo o actual frente a lo tradicional o clásico ${ }^{24}$ :

\section{3, 103 sol luna Deus $\alpha:$ sol $\beta$}

14, 117 Deus $\alpha$ : Virgilius $\beta$

14, 129 homo uir mulier $\alpha$ : dies $\beta$

15, 139 doctus est Careto doctior Fronto et doctissimus Seruandus $\alpha$ : doctus est Cato doctior Virgilius et doctissimus Donatus $\beta$

20,267 petra terra lignum et cetera $\alpha$ : petra terra paries $\beta$

23, 355-357 'Onemundus' 'o' pronomen est 'ne' coniunctio est 'mundus' nomen est $\alpha$ : 'Esidorus' 'e' praepositio est 'si' coniunctio est 'do' uerbum est 'rus' nomen est $\beta$

Proceder inexcusable en las enseñanzas del gramático antiguo y medieval era confirmar las diferentes explicaciones que ofrecía mediante exempla de auctores considerados modelos de lengua correcta y de calidad. Por esa razón los manuales de gramática están llenos de citas de esos auctores, en muchos casos heredadas de la tradición gramatical precedente, en otros casos resultado de aportación personal actualizadora. En el Ars de Julián están incluidas 713 citas y las novedosas están tomadas principalmente, como ya queda dicho ${ }^{25}$, de poetas cristianos en general y de poetas visigóticos en particular. Pero también en este campo puede intervenir algún maestro o lector experto. En 513 citas utilizadas como exempla de un total de $577^{26}$ (esto es, en el $89 \%$ de los casos) coinciden exactamente las dos líneas de transmisión. Hay, pues, 64 reproducciones de textos de auctores en las que divergen las dos familias ${ }^{27}$, bien porque

${ }^{24}$ Para que los ejemplos que cito puedan ser entendidos mejor en su contexto, considero oportuno indicar el tema que está siendo explicado respectivamente en cada caso: nombres que aluden a un solo referente (cada ejemplo va además seguido de la correspondiente justificación), nombres propios, nombres apelativos, grados del adjetivo, género no motivado naturalmente, nombres de figura compuesta múltiple.

${ }^{25}$ Véase más arriba la nota 8.

${ }^{26}$ Es el número que queda si excluimos las 80 citas correspondientes a la Conlatio de generibus metrorum, solamente conservada por la familia $\alpha$, y las 56 pertenecientes a la sección De partibus orationis II, transmitida exclusivamente por el códice $F$.

27 Por considerarlo error de transmisión, no tomo en cuenta el caso de 138,55 , donde en lugar del verso VI 70 de Juvenal, que ofrece correctamente la familia $\alpha$ para justificar las 
presentan la misma cita con alguna variación (9 casos), bien porque ofrecen exempla distintos para la misma explicación ( 9 casos), bien sobre todo porque una familia incluye alguna cita en lugares donde la otra no (46 casos).

En el grupo de variaciones en la misma cita encontramos 3 casos de variante textual. El primero de ellos se produce en el uso de un fragmento del capítulo 7 de la Vita Pauli de Jerónimo, para explicar la palabra heus $(83,111)$ :

Heus tu, inquit, quanam in parte est hic homo Dei? $\alpha$ : Heus tu, in quanam parte est hic homo Dei? $\beta$

Es posible que esa variante textual se deba a algún error por haplografía cometido en el proceso de copia. Parece, en cambio, totalmente voluntaria la modificación que aparece en el ejemplo (quizá inspirado en $I V$ Reg. 17.28) aducido para ilustrar el uso de igitur $(102,202)$ :

Igitur quum uenisset Dominus $\alpha$ : Igitur quum uenisset uir Dei $\beta$

Una situación intermedia interesante ofrece el texto del Evangelio de Lucas (1.5) citado para ejemplificar el ablativo filiabus $(27,441)$ :

Et uxor illi de filiabus Aaron $\alpha$ : Et uxor eius (ei $L$ ) de filiabus Aaron $\beta$

La variante en el texto bíblico podría deberse a un error mecánico de copia, pero es más probable que se deba a una intervención voluntaria o involuntaria motivada por la existencia de lecturas bíblicas propias de la Vetus Latina bien conocidas en la Hispania visigótica ${ }^{28}$.

Los otros 6 casos de diferencia en la misma cita consisten en que la familia $\beta$ presenta un texto más reducido que la familia $\alpha$ : en tanto que la familia $\alpha$ reproduce un texto más largo y completo, para dar entrada a lo que puede considerarse una unidad sintáctica y semántica, la familia $\beta$ ofrece una cita reducida al fragmento exclusivamente necesario para ilustrar el aspecto gramatical explicado. El resultado más llamativo es el correspondiente al capí-

características prosódicas del sustantivo subligar, la familia $\beta$ introduce el verso VI 353 del mismo Juvenal, utilizado un poco antes $(137,24)$ para ilustrar el funcionamiento prosódico del sustantivo ceruical.

${ }^{28}$ Véase Carracedo-Fraga 2014b, p. 180. La misma cita bíblica aparece también con la variante illi en la sección De partibus orationis II (193, 19 Munzi). 
tulo dedicado a explicar la posibilidad de asociar el pronombre ille a la primera persona $(40,143)$ : $\alpha$ incluye los cuatro primeros versos del poema 3 de Isidoro de Sevilla, en cambio $\beta$ reproduce solamente el primer verso.

Los casos de utilización de exempla alternativos permiten ver que en ambas familias se juega, además de con textos clásicos propios de la tradición gramatical, con textos bíblicos, litúrgicos y de poetas cristianos característicos del Ars de Julián, tal como puede comprobarse en la siguiente tabla:

\begin{tabular}{|l|l|l|}
\hline \multicolumn{1}{|c|}{ tema gramatical } & \multicolumn{1}{|c|}{$\alpha$} & \multicolumn{1}{|c|}{$\beta$} \\
\hline coniunctio - que $(37,70)$ & Gen. 4.6 & Ex. 6.2 \\
\hline pronomen iste $(41,173)$ & Hymn. Hisp. 139.1 & Breu. Mozar. (PL 86, 1020) \\
\hline pronomen hic $(42,194)$ & Incert. + Verg., Aen. I 16-17 & Hymn. Hisp. 187. 13 + Incert. \\
\hline littera $i(119,132)$ & Verg., Aen. I 119 & Verg., Aen. IV 569-570 \\
\hline littera $r(122,191)$ & Sedul., carm. Pasch. I 159 & Prud., tit. 17 \\
\hline littera $r(122,193)$ & Coripp., Iust. II 1 & Prud., tit. 1 \\
\hline diphthongon au $(129,46)$ & Drac., laud. I 206 & Hymn. Walpole 72.1 \\
\hline distinctio $(177,7)$ & Ps.Cato, dist. I 1.1-2 & Psalm. 103.24 \\
\hline periodus $(178,21)$ & Ven. Fort., carm. IX 2.1-2 & Psalm. 81.1 \\
\hline
\end{tabular}

Tabla 1: citas alternativas.

Considero interesante llamar la atención de forma expresa sobre los casos relacionados con el pronombre iste y con el diptongo au, que son respectivamente los siguientes:

Iste homo in professione sua non est praeuaricatus $\alpha$ : Iste electus Iohannes $\beta$ Aurora iam spargit polum $\alpha$ : Auroram iam quarta dies praemiserat undis $\beta$

En ambos casos el hecho de que los respectivos textos tengan el mismo comienzo tal vez pudo provocar una substitución involuntaria. De todas formas, sea cual sea la causa del cambio, está claro que los autores responsables de ambas propuestas trabajaban en un ambiente familiarizado con textos litúrgicos visigóticos y con los versos de Draconcio revisados por Eugenio de Toledo ${ }^{29}$.

29 Sobre la intervención de Eugenio en el texto de Draconcio puede verse Alberto 2005, pp. 279-390. 
Con autores y textos utilizados en la escuela en la que trabajaba Julián están también relacionadas las citas de autoridad exclusivas de cada familia. Obsérvese la siguiente tabla comparativa:

\begin{tabular}{|l|c|c|c|c|c|}
\hline \multicolumn{1}{|c|}{ AUTOR } & $\alpha$ & $\beta$ & AUTOR & $\alpha$ & $\beta$ \\
\hline Auson. & 3 & --- & Ou. & 1 & -- \\
\hline Biblia & 7 & 3 & Prud. & 5 & 2 \\
\hline Cato (Ps.) & --- & 1 & Sedul. & 1 & --- \\
\hline Coripp. & 2 & --- & Symph. & 1 & -- \\
\hline Eugen. Tol. & 2 & --- & Ven. Fort. & 2 & --- \\
\hline Hymn. Hisp. & 1 & --- & Verg. & 5 & 2 \\
\hline Incert. & 5 & 3 & TOTAL & 34 & 11 \\
\hline
\end{tabular}

Tabla 2: citas distintas.

Es evidente que el número de citas exclusivas es considerablemente superior en la rama de transmisión $\alpha$. Sin embargo, en ambas familias todos los casos de citas propias responden a las mismas tres justificaciones: se suple algún ejemplo ilustrativo donde en la otra familia no hay (21 casos en $\alpha$ y 5 $\beta)$; al ejemplo compartido se agrega algún otro complementario, generalmente precedido de et o item ( 9 casos en $\alpha$ y 2 en $\beta)^{30}$; se ofrece algún ejemplo específico para alguna explicación propia distinta (4 casos en cada familia).

También en este grupo de casos quiero prestar particular atención a uno de ellos, ya que puede dar alguna pista sobre la forma de trabajar de los responsables de intervenir en el texto. En el capítulo dedicado a explicar que las preposiciones que pueden regir tanto acusativo como ablativo se diferencian en que con el primero significan movimiento y con el segundo situación, inmediatamente después de referirse a sub con acusativo la familia $\alpha$ añade lo siguiente (107, 97): Da eius exemplum: «Stilio manibus nititur et moratur in aedibus regis». Es evidente que el texto tomado de las Sagradas Escrituras (Prou. 30.28 ) está fuera de lugar, ya que debería ir unas líneas antes $(107,94)$, para

${ }^{30}$ En varias ocasiones coinciden también las dos familias en presentar dos o tres citas de auctores para ejemplificar un único contenido, lo cual demuestra que era también un proceder del propio Julián, por otra parte, habitual en la escuela del gramático. 
ilustrar el uso de la preposición in con ablativo. Es probable que estemos ante una errónea inserción en el texto de un añadido escrito al margen.

Las intervenciones voluntarias más evidentes son aquellas en las que cada una de las dos líneas de transmisión ofrece variaciones relevantes en las explicaciones de los aspectos gramaticales objeto de estudio. Puede servir de ejemplo el primer caso que encontramos en el inicio de la obra, en el apartado dedicado a las qualitates nominum $(13,99 \text { ss. })^{31}$ :

$\alpha$

Qualitas nominum in quo est? Bipertita est. Quomodo bipertita est? Bis partita, in duabus partibus diuisa, in proprium et appellatiuum.

Quid est proprium? Quod unius est, ut 'sol', 'luna', 'Deus'. Quomodo dicis 'solem' propriae qualitatis esse, quum dicat «Soles ire et redire possunt»? Pro diebus hoc dictum est. Quomodo dicis 'lunam' propriae qualitatis esse, quum dicat 'luna prima', 'secunda', 'tertia'? Feriarum numerus hic tenetur. 'Deus' quomodo dicis propriae qualitatis esse, quum dicat "Deus deorum» et cetera? Pro principantibus hominibus dictum est.

Da appellatiuae: 'uir', 'mulier' uel 'stellae'. Quomodo? Quia multi sunt uiri, mulieres uel stellae. $\beta$

Qualitas nominum in quo est? Bipertita est. Quomodo bipertita? Bis partita, $\underline{i d}$ $\underline{\text { est }}$ in duabus partibus diuisa, in proprium et appellatiuum.

Da propriae qualitatis nomen: 'sol'. Et quia dicit «Soles ire et redire possunt»? Pro diebus hoc dixit.

Da appellatiuae: 'uir', 'mulier' uel 'stellae'. Quomodo? Quia multae sunt stellae, multi uiri et multae mulieres.

Las dos redacciones comparten: con pequeñas variantes textuales, la perícopa del Ars minor de Donato objeto de comentario $(585,10)$ y la explicación etimológica de bipertita, que coincide con la que se lee en el Ars grammatica de Cledonio $(10,21)$; con algunas variaciones en la presentación, el ejemplo sol para nombre propio, acompañado de la explicación del uso excepcional de Catulo (5.4), y los tres ejemplos de nombre común. La redacción de $\alpha$

${ }^{31}$ Resalto con subrayado el texto paralelo alternativo y con subrayado y cursiva el texto presente en una sola de las dos familias. 
por su parte añade: la definición de proprium, tomada del tratado gramatical de Pompeyo $(137,30)$, y dos ejemplos más de nombres propios con las correspondientes explicaciones para usos excepcionales (para el nombre Deus utiliza una cita de Deut. 10.17) ${ }^{32}$. ¿Está buscando el responsable de la redacción $\alpha$ completar de forma simétrica tres ejemplos en las dos partes de la explicación o es el autor de la redacción $\beta$ el que reduce?

Pero no es siempre la misma familia la que modifica y amplía explicaciones gramaticales, sino que también en este aspecto se van alternando de forma semejante las dos redacciones. Muy poco después del fragmento que acabo de comentar tenemos ya una muestra de la situación contraria a la anterior $(15,143$ ss.):

Et quot gradus sunt in conparatione? Tres. Qui? Positiuus, conparatiuus $\underline{e t}$ superlatiuus. $\beta$

Et quot gradus sunt in conparatione? Tres. Qui? Positiuus, conparatiuus, superlatiuus. Positiuus plures formas habet: exit in 'us', ut 'doctus'; exit in 'is', ut 'doctilis'; exit in 'ens', ut 'prudens'; exit in 'er', ut 'niger'. Conparatiuus duas formas habet: 'ior' et 'ius', 'doctior' et 'doctius'. Superlatiuus in singulis generibus binas: 'simus' et 'rimus', 'sima' et 'rima'. 'simum' et 'rimum'. Qualitas et quantitas quot species habent? Quattuor: ab animo, a corpore, a fortuna, a mensura; ab animo, ut 'prudens'; a corpore, ut 'candidus'; a fortuna, ut 'diues'; a mensura, ut 'longus'.

32 Para Donato uso la edición de Holtz 1981. Las referencias de Cledonio y Pompeyo remiten al vol. 5 de los Grammatici Latini. Pompeyo trabajó a finales del siglo V en el norte de África y es fuente indiscutible de Julián; véanse los trabajos clásicos del propio Holtz 1971 y 2006, pp. 109-120, y también más recientemente Lattocco 2017, la introducción de la edición de Zago 2017 y la breve presentación de Zetzel 2018, pp. 308-309. Cledonio enseñó en Constantinopla antes que Prisciano, probablemente a mediados del siglo V (véase Zetzel 2018, p. 291); con el texto del Ars grammatica a él atribuida existen algunas coincidencias en el manual de Julián, aunque no sabemos si eso se debe a uso directo o, quizá mejor, a doctrina común llegada a la escuela de Toledo por otros caminos. 
Aquí la versión $\beta$ completa la presentación de los tres grados de la comparación con dos elementos complementarios derivados de una de las fuentes principales de Julián de Toledo para la sección dedicada al nombre, las conocidas como Explanationes in Donatum atribuidas a un gramático Sergio ${ }^{33}$ : en primer lugar inserta la lista de letras finales de los tres grados (Explan. in Don. 491, 18-23); en segundo lugar añade la tipología de los adjetivos que significan calidad y cantidad, que, como se explica en la propia Ars de Julián un poco más adelante $(16,180 \mathrm{ss}$.), son los que pueden recibir grados de comparación (Explan. in Don. 491, 34-492, 2).

Otro aspecto llamativo que diferencia las dos redacciones, pero que afecta también igualmente a ambas en lugares distintos, es la utilización de las expresiones et cetera o et reliqua para omitir elementos de alguna lista enumerativa o explicaciones repetitivas que sí aparecen en la otra versión. Así, por ejemplo, en la familia $\alpha$ tenemos la declinación completa de todos los pronombres personales, posesivos, demostrativos y fóricos $(38,100-49,330)$; por su lado, la familia $\beta$ solamente ofrece los dos primeros casos (nominativo y genitivo) para la mayor parte de esos pronombres ${ }^{34}$. Por el contrario, las largas listas de conjunciones racionales $(100,159-161)$ y de preposiciones de acusativo y ablativo con sus respectivos ejemplos $(104,33-106,75)$ están completas en $\beta$, pero en $\alpha$ aparecen reducidas solamente a algunas de las primeras de las respectivas series ${ }^{35}$.

Las diferencias de redacción entre las dos familias son especialmente abundantes en los capítulos dedicados al nombre, al pronombre, a la inter-

${ }^{33}$ Un texto incompleto de las Explanationes puede leerse en el volumen 4 de los Grammatici Latini, pp. 486-565. Las coincidencias del tratado gramatical de Julián con las Explanationes son numerosas y muchas de ellas literales. Parece claro, pues, que estamos ante una utilización directa o ante un uso de materiales comunes a ambas obras. Por su estrecha relación con el Ars de Pompeyo, se ha propuesto situar el origen de las Explanationes también en el norte de África en los siglos V o VI. Véanse los trabajos de De Paolis 2000 y 2017, la presentación resumida de Zetzel 2018, pp. 321-322, y para la relación con la Hispania visigótica Carracedo-Fraga 2014a, pp. 73-74.

${ }^{34}$ La redacción $\beta$ incluye la declinación completa de los pronombres ego y $t u$, los tres primeros casos para los pronombres tuus y suus, y los cuatro primeros casos para los pronombres is y meus.

${ }^{35}$ La familia $\alpha$ enumera solamente 4 de las 17 conjunciones racionales, 2 de las 30 preposiciones de acusativo y 8 de ellas con ejemplo, 7 de las 15 preposiciones de ablativo y ninguna de ellas con ejemplo. 
jección, a la sílaba, a los metros y a las positurae. En las dos versiones el estilo de redacción es similar al que encontramos en toda el Ars grammati$c a$ y las fuentes utilizadas para las modificaciones son las mismas de las que dispuso Julián para el proyecto originario: principalmente las ya mencionadas, Pompeyo y las Explanationes in Donatum, y además el tratado gramatical conocido con el título Excerpta de Scauri et Palladii libris y puesto bajo el nombre de Áudax ${ }^{36}$, y las Etymologiae de Isidoro de Sevilla.

\section{Conclusiones}

En la escuela de Toledo dirigida por Julián fue puesto por escrito, probablemente entre los años 680-690, un completo manual de gramática que recogía las enseñanzas elementales $\mathrm{y}$, sobre todo, avanzadas del maestro, entre las que era objeto de especial atención la métrica y la creación poética. Es probable que ese proyecto inicial, como producto escolar que era, ya incluyese propuestas alternativas o variantes de autor para algunos contenidos. Muy poco tiempo después de la redacción primigenia alguna copia de un ejemplar que ya estaba fuera de la supervisión directa de Julián ${ }^{37}$ fue utilizada en alguna escuela también visigótica (pudo ser la misma en la que trabajó el obispo toledano o una muy próxima) por algún otro maestro o por algún alumno versado, el cual procedió a introducir algunas modificaciones siguiendo las propias enseñanzas de Julián o utilizando sus mismos materiales y provocó que hoy tengamos una clara doble redacción en algunos de los contenidos del Ars grammatica, sobre todo en los apartados correspondientes al Ars minor o al libro primero del Ars maior de Donato.

Teniendo en cuenta que el resultado textual en las dos líneas de transmisión presenta características muy similares en estilo de redacción, uso de fuentes e introducción de ejemplos, resulta muy difícil poder saber si una de las dos versiones es la original o si hubo manipulación en las dos ramas de transmisión y, en ese caso, una de ellas conserva un texto más próximo al primigenio. Los aspectos que podríamos utilizar como argumentos a favor de alguna de las

${ }^{36}$ Es igualmente poco lo que sabemos de este gramático y también para él se conjetura como lugar de trabajo el norte de África y como momento de actividad la segunda mitad del siglo V o la primera del VI. Véase Carracedo-Fraga 2014a, pp. 74-75, y Zetzel 2018, pp. 283-284.

${ }^{37}$ Más arriba he querido probar la existencia de un arquetipo que contenía ya algunos errores que difícilmente pueden ser responsabilidad del obispo toledano. 
hipótesis están presentes de manera semejante en las dos redacciones: tendencia a ampliar (o a reducir) explicaciones, ejemplos ilustrativos y paradigmas flexivos; afán por utilizar como exempla palabras o textos más actualizados y novedosos en lugar de otros más clásicos y tradicionales ${ }^{38}$; existencia de elementos aparentemente mal ubicados en el conjunto que pueden ser el resultado de anotaciones marginales o interlineales inseridas en el texto.

Creo, pues, más probable que las dos redacciones son el resultado de reelaboraciones posteriores del producto primitivo, algo muy habitual en los textos escolares de gramática. Considero, en cambio, casi seguro que esas intervenciones se produjeron, como acabo de decir, en un momento muy temprano entre el estadio escrito primero del Ars grammatica y la copia de los ejemplares $\alpha$ y $\beta$, ya que los efectos resultantes están casi todos perfectamente integrados y consolidados en las copias derivadas en cada una de las dos ramas de transmisión textual. Por consiguiente, ante la imposibilidad de poder precisar o definir con claridad orden y grados de responsabilidad en el proceso de manipulación, entiendo que el editor del Ars grammatica de Julián debe presentar en paralelo las dos redacciones como frutos más o menos directos e inmediatos por igual de la labor escolar del maestro toledano.

\section{BIBLIOGRAFÍA}

Alberto, P. F. (2005): Eugenii Toletani opera omnia, Turnhout, Brepols (= CCSL 114).

Alberto, P. F. (2016): «Poésie wisigothique dans l'exemplication du Liber Glossarum», en Le Liber glossarum (s. VII-VIII): Composition, sources, réception. Dossiers d'HEL 10, pp. 159-176.

Alberto, P. F. (2017): «Poesía visigótica y escuela carolingia», en Mesa Sanz, J. (ed.), Latinidad medieval hispánica, Florencia, SISMEL-Edizioni del Galluzzo, pp. 27-53.

Alberto, P. F. (2018): «New Evidence for Julian of Toledo's Ars grammatica», Revue d'Histoire des Textes 13, pp. 165-183.

${ }^{38} \mathrm{Me}$ refiero a lo comentado sobre las variaciones en las citas de auctores o en el uso de ejemplos gramaticales. Como muestra, recuérdese que en el capítulo sobre el comparativo (15, 139-140) la redacción $\alpha$ utiliza los nombres Careto, Fronto y Seruandus, donde la versión $\beta$ presenta los nombres Cato, Virgilius y Donatus, mientras que a su vez un poco más adelante a propósito del caso nominativo $(24,372-372) \beta$ juega con los nombres Aetherius, Iohannis y Eruigius rex, y $\alpha$ solamente con el sustantivo magister. 
Becker, G. (1885): Catalogi bibliothecarum antiqui, Bonn, Cohen (reimp. Hildesheim, Olms, 1973).

Beeson, Ch. H. (1924): «The Ars grammatica of Julian of Toledo», en Miscellanea Francesco Ehrle, Roma, Biblioteca Apostolica Vaticana, vol. I, pp. 50-70.

Bischoff, B. (1989): Die Abtei Lorsch im Spiegel ihrer Handschriften, Lorsch, Laurissa.

Bischoff, B. (1998): Katalog der festländischen Handschriften des neuten Jahrhunderts (mit Ausnahme der wisigotischen), vol. 1, Wiesbaden, Harrassowitz.

Brummer, J. (1912): Vitae Vergilianae, Leipzig, Teubner.

Carracedo-Fraga, J. (2005a): «Poesía y poetas en la escuela visigótica», en Díaz y Díaz, M. C. y Díaz de Bustamante, J. M. (eds.), Poesía latina medieval (siglos $V-X V)$. Actas del IV Congreso del «Internationales Mittellateinerkomitee», Florencia, SISMEL-Edizioni del Galuzzo, pp. 93-107.

Carracedo-Fraga, J. (2005b): «Sobre la autoría del tratado gramatical atribuido a Julián de Toledo», Euphrosyne 33, pp. 189-200.

Carracedo-Fraga, J. (2014a): «De gramáticas y gramáticos en la Hispania visigótica», en Codoñer, C. y Alberto, P. F. (eds.), Wisigothica. After M. C. Díaz y Díaz, Florencia, SISMEL-Edizioni del Galuzzo, pp. 67-89.

Carracedo-Fraga, J. (2014b): «La Biblia en el Ars grammatica de Julián de Toledo», en Ruiz Arzalluz, I. (ed.), Estudios de filología e historia en honor del profesor Vitalino Valcárcel, Vitoria, Servicio Editorial Universidad del País Vasco, vol. 1, pp. 169-182.

Carracedo-Fraga, J. (2015): El tratado «De uitiis et uirtutibus orationis» de Julián de Toledo. Estudio, edición y traducción, Santiago de Compostela, USC-Editora.

Carracedo-Fraga, J. (2018): «Problemas y soluciones en la edición de un tratado gramatical de testimonio único: el De partibus orationis de Julián de Toledo», Filologia Mediolatina 25, pp. 83-111.

Carracedo-Fraga, J. (2020): «Isidore of Seville as a Grammarian», en Fear, A. y Wood, J. (eds.), A Companion to Isidore of Seville, Leiden, Brill, pp. 222-244.

Castro Sánchez, J. (2010): Hymnodia Hispanica, Turnhout, Brepols (= CCSL 167).

Christ, K. (1933): Die Bibliothek des Klosters Fulda im 16. Jahrhundert. Die Handschriften-Verzeichnisse, Leipzig, Harrassowitz.

De Paolis, P. (2000): «Le Explanationes in Donatum e il loro più antico testimone manoscritto», en De Nonno, M., De Paolis, P. y Holtz, L. (eds.), Manuscripts and Tradition of Grammatical Texts from Antiquity to the Renaissance, Cassino, Università degli Studi di Cassino, vol. 1, pp. 173-221.

De Paolis, P. (2017): «Le strategie linguistiche e didattiche dei commenti a Donato: osservazioni sulle Explanationes in Donatum», en García Leal, A. y Prieto Entrialgo, C. E. (eds.), Latin vulgaire-latin tardif XI, Hildesheim, Olms, pp. 672-683. 
Funaioli, G. (1911): «Su Giuliano Toletano», Rivista di filologia e istruzione classica 39, pp. 42-79.

Holtz, L. (1971): «Tradition et diffusion de l'oeuvre grammaticale de Pompée, commentateur de Donat», Revue de philologie 45, pp. 48-83.

Holtz, L. (1981): Donat et la tradition de l'enseignement grammatical. Étude sur l'Ars Donati et sa diffusion (IVe-IXe siècle) et édition critique, París, CNRS (reimp. 2010).

Holtz, L. (2006): «Prolégomènes à une édition critique du commentaire de Pompée, grammarien Africain», en Taifacos, I. (ed.), The Origins of European Scholarship, Stuttgart, Franz Steiner, pp. 109-120.

Irvine, M. (1994): The Making of Textual Culture. "Grammatica» and Literary Theory 350-1100, Cambridge, Cambridge University Press.

Lattocco, A. (2017): «L'esegesi grammaticale in Giuliano e in Pompeo vs Donato», Atene e Roma 3-4, pp. 140-147.

Law, V. (1997): Grammar and Grammarians in the Early Middle Ages, Londres, Logman.

Lorenzana, F. de (1797): Sancti Iuliani episcopi Toletani Ars grammatica, poetica et rhetorica e membranis antiquis Bibliothecae Vaticano-Palatinae nunc primum in lucem edita, Roma, apud Antonium Fulgonium.

Maestre Yenes, M. A. H. (1973): Ars Iulani Toletani episcopi: una gramática latina de la España visigoda, Toledo, Publicaciones del Institituto Provincial de Investigaciones y Estudio Toledanos.

Mostert, M. (1989): The Library of Fleury: a Provisional List of Manuscripts, Hilversum, Verloren Publishers.

Munzi, L. (1980-1981): «Il De partibus orationis di Giuliano di Toledo», Annali dell'Istituto Universitario Orientale di Napoli, Seminario di Studi del Mondo Classico, Sezione filologico-letteraria 2-3, pp. 153-228.

Pérez de Urbel, J. y González y Ruiz-Zorrilla, A. (1950): Liber Commicus, Madrid, CSIC. Spevak, O. (2020): Isidore de Séville. Etymologies, livre I: la grammaire. Texte établi, traduit et commenté par O. S., París, Les Belles Lettres.

Zago, A. (2017): Pompeii Commentum in Artis Donati partem tertiam. Introduzione, testo critico e commento, Hildesheim, Weidmann.

Zetzel, J. E. G. (2018): Critics, Compilers, and Commentators: an Introduction to Roman Philology, 200 BCE-800 CE, Oxford, Oxford University Press.

Fecha de recepción de la primera versión del artículo: 15/06/2020

Fecha de aceptación: 25/09/2020

Fecha de recepción de la versión definitiva: 09/11/2020 